\title{
Infimum of the spectrum of Laplace-Beltrami operator on a bounded pseudoconvex domain with a Kähler metric of Bergman type
}

\author{
SONG-YING Li AND MY-An TRAN
}

\begin{abstract}
The research in paper is a continuation of the work of Li and Wang [10-12] who studied upper estimates for $\lambda_{1}=\lambda_{1}\left(\Delta_{g}\right)$, the bottom of the spectrum of Laplace-Beltrami operator on a complete noncompact Kähler manifold $\left(M^{n}, g\right)$ with a lower bound condition on holomorphic bisectional curvature and the work of Munteanu [16] who uses lower bound condition on Ricci curvature. In this paper, we study the problems on a bounded pseudoconvex domain $D$ in $\mathbb{C}^{n}$ with a certain normalized complete Kähler metrics $u$ on $D$ which are called Bergman-type, we find a class of Bergman-type metrics $u$ on $D$ so that $\lambda_{1}\left(\Delta_{u}\right)=n^{2}$. We also provide a simple condition on metric $u$, under this condition, we obtain the sharp upper bound estimates $n^{2}$ for $\lambda_{1}\left(\Delta_{u}\right)$ for such class of Bergmantype metrics, which include Kähler-Einstein metric and Bergman metric on $D$.
\end{abstract}

\section{Introduction}

Let $\left(M^{n}, g\right)$ be a Kähler manifold of dimension $n$ with Kähler metric $g=$ $\sum_{i, j=1}^{n} g_{i \bar{j}} d z_{i} \otimes d \bar{z}_{j}$. Let

$$
\Delta_{g}=-4 \sum_{i, j=1}^{n} g^{i \bar{j}} \frac{\partial^{2}}{\partial z_{i} \partial \bar{z}_{j}}
$$

be the Laplace-Beltrami operator with respect to the metric $g$, where $\left[g^{i \bar{j}}\right]^{t}=$ $\left[g_{i \bar{j}}\right]^{-1}$. Let

$$
\lambda_{1}\left(\Delta_{g}, M\right)=\inf \left\{4 \int_{M} \sum_{i, j=1}^{n} g^{i \bar{j}} \frac{\partial f}{\partial z_{i}} \frac{\partial f}{\partial \bar{z}_{j}} d V_{g}: f \in C_{0}^{\infty}(M),\|f\|_{L^{2}}=1\right\},
$$

where $d V_{g}$ is the volume measure on $M$ with respect to the Kähler metric $g$. 
When $M$ is compact and $\Delta_{g}$ is uniformly elliptic, $\lambda_{1}\left(\Delta_{g}\right)$ is the first positive eigenvalue of $\Delta_{g}$ with Dirichlet boundary condition. A lot of research has been done on its upper and lower bound estimates and its impact on geometry and physics (see, for example, the lecture notes of $\mathrm{Li}$ [8] and the paper of Udagawa [17] and references therein).

When $\left(M^{n}, g\right)$ is a complete non-compact Kähler manifold, $\lambda_{1}\left(\Delta_{g}\right)$ may not be an eigenvalue of $\Delta_{g}$. For example, when $M$ is the complex hyperbolic space $\mathbb{C H}^{n}, \lambda_{1}\left(\Delta_{g}\right)$ is no longer an $L^{2}$ eigenvalue of $\Delta_{g}$. However, it is the infimum of the positive spectrum of $\Delta_{g}$. The problem of finding estimates for $\lambda_{1}\left(\Delta_{g}\right)$ in the complete non-compact case has been studied by many mathematicians. An important upper bound estimate was obtained by Li and Wang [10]. With the assumption that the holomorphic bisectional curvature of $M$ is bounded below by -1 , they proved that $\lambda_{1}\left(\Delta_{g}\right) \leq n^{2}$. Their estimate is sharp and equality is achieved by the complex hyperbolic space form $\mathbb{C H}^{n}$. O. Munteanu obtained another estimate in [16], where he proved that $\lambda_{1}\left(\Delta_{g}\right)$ is bounded from above by $n^{2}$ if the Ricci curvature of $M$ is bounded from below by $-2(n+1)$ (or $\left.R_{i \bar{j}} \geq-(n+1) g_{i \bar{j}}\right)$. His estimate is also sharp and equality is achieved by the complex hyperbolic space form $\mathbb{C H}^{n}$. On the other hand, the precise information on $\lambda_{1}$ can be used to deduce information on the geometry of manifolds. Along this line, many works have been done by Li, Wang, Ji, Kong, Zou, and several other authors (see [5, 6, 10-12], and references therein).

The main purpose of this note is to provide more examples of complete Kähler manifolds for which the precise value of $\lambda_{1}$ can be computed. We consider a bounded pseudoconvex domain $D$ in $\mathbb{C}^{n}$ with a Kähler metric $u_{i \bar{j}} d z_{i} \otimes d \bar{z}_{j}$, where $u_{i \bar{j}}=\frac{\partial^{2} u}{\partial z_{i} \partial \bar{z}_{j}}$ with $u$ being a strictly plurisubharmonic exhaustion function for $D$. If $D$ is $B_{n}$, the unit ball in $\mathbb{C}^{n}$, and $u(z)=-\log \left(1-|z|^{2}\right)$, then $u_{i j} d z_{i} \otimes d \bar{z}_{j}$ is both the Bergman metric and the Kähler-Einstein metric on $B_{n}$. To find the exact value of $\lambda_{1}\left(\Delta_{u}\right)$ on $B_{n}$, one approach is to estimate both the upper bound and the lower bound. We first let $f(z)=\left(1-|z|^{2}\right)^{n / 2}$. To obtain the upper bound, we apply the Rayleigh's principle, which gives $\lambda_{1} \leq \int_{B_{n}}|\nabla f|^{2} / \int_{B_{n}}|f|^{2}=n^{2}$. To obtain the lower bound, we apply Proposition 9.2 in [9], which states that $\lambda_{1} \geq \mu>0$ if there exists a positive function $h$ such that $\Delta_{u} h \geq \mu h$. In fact, the function $f$ defined above satisfies $\Delta_{u} f \geq n^{2} f$. This implies that $\lambda_{1}\left(\Delta_{u}\right)=n^{2}$ on $B_{n}$. For a general bounded pseudoconvex domain $D$, the situation is more complicated and, therefore, more subtle arguments are required to obtain the exact value of $\lambda_{1}\left(\Delta_{u}\right)$. We will impose various conditions on the exhaustion function $u$ on $D$. Under these conditions, we will estimate the upper and lower bounds for $\lambda_{1}$ by constructing special functions and carrying out the analysis on a specific subdomain of $D$. 
Let $D$ be a bounded pseudoconvex domain in $\mathbb{C}^{n}$ with $C^{2}$ boundary. Let $r \in C^{2}\left(\mathbb{C}^{n}\right)$ be a defining function for $D$ so that $u(z)=-\log (-r(z))$ is strictly plurisubharmonic in $D$. Then the complete Kähler metric induced by $u$ is

$$
u=\sum_{i, j=1}^{n} u_{i j} d z_{i} \otimes d \bar{z}_{j}
$$

Let

$$
|\partial u|_{u}^{2}=\sum_{i, j=1}^{n} u^{i \bar{j}} \partial_{i} u \partial_{\bar{j}} u
$$

where $\left[u^{i \bar{j}}\right]^{t}=\left[u_{i \bar{j}}\right]^{-1}$. Let

$$
\beta(z)=\limsup _{w \rightarrow z}|\partial u(w)|_{u}^{2}, \quad z \in \partial D
$$

We will prove the following:

Theorem 1.1. Let $D$ be a bounded pseudoconvex domain in $\mathbb{C}^{n}$ with a defining function $r(z) \in C^{2}\left(\mathbb{C}^{n}\right)$. Assume that $u(z)=-\log (-r(z))$ is strictly plurisubharmonic in $D$ with $\beta(z)=1$ on $\partial D$. Then, with the notation $\lambda_{1}(D)$ $=\lambda_{1}\left(\Delta_{u}, D\right)$, the following statements hold:

(a) $\lambda_{1}(D) \leq \lambda_{1}(D \backslash K) \leq n^{2}$ for any compact subset $K$ of $D$;

(b) If, in addition, $r(z)$ is plurisubharmonic in $D$, then $\lambda_{1}(D)=n^{2}$.

Corollary 1.1. Let $D$ be a smoothly bounded strictly pseudoconvex domain in $\mathbb{C}^{n}$ with defining function $r(z)$ and $u(z)=-\log (-r(z))$. Then

(i) If $r$ is strictly plurisubharmonic in $D$, then $\lambda_{1}(D)=n^{2}$,

(ii) If $\sum_{\alpha, \beta=1}^{n} u_{\alpha \bar{\beta}} d z_{\alpha} \otimes d \bar{z}_{\beta}$ is the Kähler-Einstein metric in D, then $\lambda_{1}$ $\left(\Delta_{u}, D\right) \leq n^{2}$, where $u$ is the strictly plurisubharmonic solution of Monge-Ampère equation:

$$
\operatorname{det} H(u)=\mathrm{e}^{(n+1) u}, \text { in } D ; \quad u=\infty \text { on } \partial D
$$

(iii) If $\sum_{\alpha, \beta=1}^{n} u_{\alpha \bar{\beta}} d z_{\alpha} \otimes d \bar{z}_{\beta}$ is the Bergman metric on $D$, then $\lambda_{1}\left(\Delta_{u}, D\right)$ $\leq n^{2}$, where

$$
u_{i \bar{j}}=\frac{1}{n+1} \frac{\partial^{2} \log K(z, z)}{\partial z_{i} \partial \bar{z}_{j}}
$$


and $K(z, w)$ is the Bergman kernel function for the domain $D$.

Remark 1.1. (a) Part (ii) of Corollary 1.2 was proved by Munteanu in [16], we provide an alternate proof here,

(b) The condition $\beta(z)=1$ on $\partial D$ is an analysis condition, but $\beta(z)=1$ near $\partial D$, has geometric interpretation related to pseudo scalar curvature for Kähler-Einstein metric, see [13, 15] for the detail.

This paper is organized as follows: In Section 2, we will prove several theorems for a bounded pseudoconvex domain $D$ in $\mathbb{C}^{n}$ with a strictly plurisubharmonic exhaustion function $u$ that satisfies various conditions. As a consequence of those results, we will prove Theorem 1.1 there. Corollary 1.2 will be proved in Section 3. Finally, in Section 4, we will provide examples of weakly (not strictly) pseudoconvex domains for which $\lambda_{1}=n^{2}$. Specifically, we consider the complex ellipsoid $E_{m}=\left\{z=\left(z_{1}, z_{2}\right) \in \mathbb{C}^{2}: r(z)<0\right\}$, where $r(z)=\left|z_{2}\right|^{2}-\left(1-\left|z_{1}\right|^{2}\right)^{1 / m}$ and $m>1$. With $u(z)=-\log (-r(z))$, we will prove that $\lambda_{1}\left(\Delta_{u}, E_{m}\right)=4=2^{2}$.

\section{Preliminary setting and main theorems}

Let $D$ be a bounded pseudoconvex domain in $\mathbb{C}^{n}$ with a defining function $r \in C^{2}\left(\mathbb{C}^{n}\right)$ so that $u(z)=-\log (-r(z))$ is strictly plurisubharmonic in $D$. We consider the Laplace-Beltrami operator $\Delta_{u}$ associated to the Kähler metric $u_{i j} d z_{i} \otimes d \bar{z}_{j}$ on $D$, which is given by

$$
\Delta_{u}=-4 \sum_{i, j=1}^{n} u^{i \bar{j}} \frac{\partial^{2}}{\partial z_{i} \partial \bar{z}_{j}}
$$

where $\left[u^{i \bar{j}}\right]^{t}=H(u)^{-1}=\left[u_{i \bar{j}}\right]^{-1}$.

We start with the following lemma.

Lemma 2.1. Let $\Omega$ be any domain in $\mathbb{C}^{n}$, and let $u_{i \bar{j}} d z_{i} \otimes d \bar{z}_{j}$ be any Kähler metric on $\Omega$, where $u_{i \bar{j}}=\partial_{i \bar{j}} u$ and $u \in C^{2}(\Omega)$ is strictly plurisubharmonic. Let $f(z)=\mathrm{e}^{-\alpha u(z)}$ in $\Omega$ for some $\alpha>0$. Then the following statements hold:

(i) The Laplacian of $f$ is given by

$$
\Delta_{u} f(z)=4 \alpha f(z)\left(n-\alpha|\partial u|_{u}^{2}\right)
$$


Laplace-Beltrami operator on a bounded pseudoconvex domain 379 where

$$
|\partial u|_{u}^{2}=\sum_{i, j=1}^{n} u^{i \bar{j}} u_{i} u_{\bar{j}}=\sum_{i, j=1}^{n} u^{i \bar{j}} \partial_{i} u \partial_{\bar{j}} u
$$

(ii) If $r(z)=-\mathrm{e}^{-u(z)}$ is plurisubharmonic, then $|\partial u|_{u}^{2} \leq 1$ on $\Omega$,

(iii) Suppose that $\Omega$ is bounded with $\partial \Omega \in C^{1}$. Let $h_{1}, h_{2} \in C^{2}(\Omega) \cap C^{1}(\bar{\Omega})$. Then

$$
\begin{aligned}
\int_{\Omega} & \left(h_{2} \Delta_{u} h_{1}-h_{1} \Delta_{u} h_{2}\right) d V_{u} \\
=\int_{\partial \Omega} & {\left[h_{2}\left(-\sum_{i, j=1}^{n} u^{i \bar{j}} \nu_{i} \frac{\partial h_{1}}{\partial \bar{z}_{j}}\right)\right.} \\
& \left.-h_{1}\left(-\sum_{i, j=1}^{n} u^{i \bar{j}} \nu_{\bar{j}} \frac{\partial h_{2}}{\partial z_{i}}\right)\right] g(z) d \sigma(z) .
\end{aligned}
$$

In particular, if $\Delta_{u} h_{1}(z) \geq 0$ in $\Omega, h_{1}(z)=0$ on $\partial \Omega$, and $h_{2}(z) \geq 0$ on $\partial \Omega$, then

$$
\int_{\Omega}\left(h_{2} \Delta_{u} h_{1}-h_{1} \Delta_{u} h_{2}\right) d V_{u} \geq 0
$$

Here,

$$
g(z)=\operatorname{det} H(u) \quad \text { and } \quad d V_{u}(z)=g(z) d v(z),
$$

and $\nu(z)=\left(\nu_{1}(z), \ldots, \nu_{n}(z)\right)$ is the complex outward normal vector to $\partial \Omega$ so that $|\nu(z)|^{2}=4^{2}$.

Proof. Note that

$$
\begin{aligned}
\Delta_{u} f(z) & =-4 f(z) \sum_{i, j=1}^{n} u^{i \bar{j}}\left[-\alpha u_{i \bar{j}}+\alpha^{2} u_{i} u_{\bar{j}}\right] \\
& =4 \alpha f(z)\left[n-\alpha|\partial u|_{u}^{2}\right] .
\end{aligned}
$$

Thus (i) follows. Next we prove (ii). Straightforward computation shows that

$$
u_{i \bar{j}}=\frac{1}{-r}\left[r_{i \bar{j}}+\frac{1}{-r} r_{i} r_{\bar{j}}\right], \quad u^{i \bar{j}}=(-r)\left[r^{i \bar{j}}-\frac{r^{i} r^{\bar{j}}}{|\partial r|_{r}^{2}-r}\right],
$$


where

$$
|\partial r|_{r}^{2}=\sum_{i, j=1}^{n} r^{i \bar{j}} r_{i} r_{\bar{j}}, \quad r^{i}=\sum_{j=1}^{n} r^{i \bar{j}} r_{\bar{j}} \text { and } r^{\bar{j}}=\sum_{i=1}^{n} r^{i \bar{j}} r_{i}
$$

Thus, since $|\partial r|_{r}^{2} \geq 0$, one has

$$
|\partial u|_{u}^{2}=\frac{1}{-r}\left[|\partial r|_{r}^{2}-\frac{|\partial r|_{r}^{4}}{|\partial r|_{r}^{2}-r}\right]=\frac{|\partial r|_{r}^{2}}{|\partial r|_{r}^{2}-r} \leq 1
$$

Finally, by the Divergence Theorem in complex coordinates and the fact that $\sum_{i=1}^{n} \partial_{i}\left(u^{i \bar{j}} g\right)=\sum_{j=1}^{n} \partial_{\bar{j}}\left(u^{i \bar{j}} g\right)=0$, we have

$$
\begin{aligned}
& \int_{\Omega}\left(h_{2} \Delta_{u} h_{1}-h_{1} \Delta_{u} h_{2}\right) d V_{u} \\
&=\int_{\Omega}\left[\sum_{i=1}^{n} \frac{\partial}{\partial z_{i}}\left(h_{2}\left(-\sum_{j=1}^{n} u^{i \bar{j}} g \frac{\partial h_{1}}{\partial \bar{z}_{j}}\right)\right)\right. \\
&\left.-\sum_{j=1}^{n} \frac{\partial}{\partial \bar{z}_{j}}\left(h_{1}\left(-\sum_{i=1}^{n} u^{i \bar{j}} g \frac{\partial h_{2}}{\partial z_{i}}\right)\right)\right] d v \\
&=\int_{\partial \Omega}\left[h_{2}(z)\left(-\sum_{i, j=1}^{n} u^{i \bar{j}} \nu_{i} \frac{\partial h_{1}}{\partial \bar{z}_{j}}\right)\right. \\
&\left.-h_{1}(z)\left(-\sum_{i, j=1}^{n} u^{i \bar{j}} \nu_{\bar{j}} \frac{\partial h_{2}}{\partial z_{i}}\right)\right] g(z) d \sigma(z) .
\end{aligned}
$$

Thus, (2.4) holds. If $\Delta_{u} h_{1} \geq 0$ in $\Omega$ and $h_{1}=0$ on $\partial \Omega$, by the Maximum Principle, we have $-\sum_{i, j=1}^{n} u^{i \bar{j}} \nu_{i} \frac{\partial h_{1}}{\partial \bar{z}_{j}} \geq 0$ on $\partial \Omega$. By the assumption $h_{2} \geq 0$ on $\partial \Omega$ and (2.4), we have that (2.5) holds. Therefore, the proof of part (iii) is complete, and so is the proof of the lemma.

As a corollary of the previous lemma and a proposition proved by $\mathrm{Li}$ (see Proposition 9.2 in [9]), one has

Proposition 2.1. Let $\Omega$ be any domain in $\mathbb{C}^{n}$ and let $u \in C^{2}(\Omega)$ be strictly plurisubharmonic. If

$$
|\partial u|_{u}^{2} \leq \beta \quad \text { in } \Omega
$$


Laplace-Beltrami operator on a bounded pseudoconvex domain 381

for some constant $\beta>0$, then

$$
\lambda_{1}\left(\Delta_{u}, \Omega\right) \geq \frac{n^{2}}{\beta}
$$

where $\lambda_{1}\left(\Delta_{u}, \Omega\right)$ is the infimum of the positive spectrum of $\Delta_{u}$ on $\Omega$.

Proof. Let $f(z)=\mathrm{e}^{-\alpha u(z)}$. By Lemma 2.1, one has

$$
\Delta_{u} f(z)=4 \alpha\left(n-\alpha|\partial u|_{u}^{2}\right) f(z) \geq 4 \alpha(n-\alpha \beta) f(z), \quad z \in \Omega
$$

By the argument of the proposition of $\operatorname{Li}[9]$ on $\Omega$, one has that

$$
\lambda_{1}\left(\Delta_{u}, \Omega\right) \geq 4 \alpha(n-\alpha \beta), \quad \alpha>0 .
$$

In fact, for any $\epsilon>0$, let $\Omega_{\epsilon} \subset \Omega$ be a compact subdomain of $\Omega$ such that $\partial \Omega_{\epsilon} \in C^{\infty}$ and $\Omega_{\epsilon} \uparrow \Omega$ as $\epsilon \rightarrow 0^{+}$. Let $\lambda_{1}(\epsilon)$ be the first positive eigenvalue for the Dirichlet problem for $\Delta_{u}$ with the eigenfunction $v(z)$ on $\Omega_{\epsilon}$. Then the regularity of $v$ implies that $v$ is positive in $\Omega_{\epsilon}$. Furthermore, $v=0$ on $\partial \Omega_{\epsilon}$. By (2.5) with $h_{1}=v$ and $h_{2}=f$, we have

$$
\left(\lambda_{1}(\epsilon)-4 \alpha(n-\alpha \beta)\right) \int_{\Omega_{\epsilon}} v(z) f(z) d V_{u}(z) \geq 0 .
$$

Thus $\lambda_{1}(\epsilon) \geq 4 \alpha(n-\alpha \beta)$ and

$$
\lambda_{1}\left(\Delta_{u}, \Omega\right)=\inf _{\epsilon} \lambda_{1}(\epsilon) \geq 4 \alpha(n-\alpha \beta) .
$$

We know that $4 \alpha(n-\alpha \beta)$ takes its maximum at $\alpha=\frac{n}{2 \beta}$. Therefore,

$$
\lambda_{1}\left(\Delta_{u}, \Omega\right) \geq 4 \frac{n}{2 \beta}\left(n-\frac{n}{2}\right)=\frac{n^{2}}{\beta}
$$

and the proof of the proposition is complete.

Notations. For convenience, we will use $\lambda_{1}\left(\Delta_{u}, D\right), \lambda_{1}(D)$, and $\lambda_{1}$ interchangeably to denote the infimum of the positive spectrum of $\Delta_{u}$ on $D$. We let $\alpha$ and $\beta$ denote positive constants. In addition, $d V_{u}$ is the volume measure on $D$ with respect to the Kähler metric $u_{i \bar{j}} d z_{i} \otimes d \bar{z}_{j}, d v$ is the Lebesgue volume measure on $\mathbb{C}^{n}$, and $d \sigma$ is the Hausdorff measure on any hypersurface in $D$. 
Let $J$ be the Fefferman operator defined in [3]. Then

$$
J(r)=-\operatorname{det}\left[\begin{array}{cc}
r & \bar{\partial} r \\
(\bar{\partial} r)^{*} & H(r)
\end{array}\right]
$$

where

$$
\bar{\partial} r=\left[r_{\overline{1}}, \ldots, r_{\bar{n}}\right], \quad(\bar{\partial} r)^{*}=\left[r_{1}, \ldots, r_{n}\right]^{t}, \quad \text { and } \quad H(r)=\left[r_{i \bar{j}}\right]
$$

We shall prove the following:

Theorem 2.2. If $|\partial u|_{u}^{2} \leq \beta$ on $D$ and $r \in C^{2}(D) \cap C^{0,1}(\bar{D})$ with $J(r)$ being bounded on $D$, then

$$
\lambda_{1}(D) \leq \beta n^{2}
$$

Proof. It is known [13] that

(2.17) $\operatorname{det} H(u)=J(r)\left(\frac{1}{-r}\right)^{n+1}, \quad d V_{u}=\operatorname{det} H(u) d v=\frac{J(r)}{(-r)^{n+1}} d v$.

Let $\alpha=\frac{n}{2}+\epsilon$ with $\epsilon>0$ very small and $f(z)=(-r(z))^{\alpha}$. Then

$$
\begin{aligned}
\int_{D}|f(z)|^{2} d V_{u}=\int_{D} \frac{(-r(z))^{n+2 \epsilon} J(r)}{(-r(z))^{n+1}} & d v \\
& =\int_{D} \frac{J(r)(z)}{(-r(z))^{1-2 \epsilon}} d v(z)<\infty
\end{aligned}
$$

since $(-r(z)) \approx \operatorname{dist}(z, \partial D)$, the (Euclidean) distance of $z$ to $\partial D$, when $z$ is near $\partial D$. Then

$$
\begin{aligned}
\lambda_{1} & \leq \frac{(\nabla f, \nabla f)_{u}}{(f, f)_{u}} \\
& =\frac{\int_{D} 4 \sum_{i, j=1}^{n} u^{i \bar{j}} \partial_{i} f \partial_{\bar{j}} f d V_{u}}{(f, f)_{u}}
\end{aligned}
$$


Laplace-Beltrami operator on a bounded pseudoconvex domain 383

$$
\begin{aligned}
& =4 \alpha^{2} \frac{\int_{D}(-r)^{2 \alpha}|\partial u|_{u}^{2} d V_{u}}{(f, f)_{u}} \\
& =4 \alpha^{2} \frac{\int_{D}|f(z)|^{2}|\partial u|_{u}^{2} d V_{u}}{(f, f)_{u}} \\
& \leq 4 \alpha^{2} \beta \frac{\int_{D}(-r)^{2 \alpha} d V_{u}}{(f, f)_{u}} \\
& =4 \alpha^{2} \beta .
\end{aligned}
$$

Letting $\alpha=\frac{n}{2}+\epsilon \rightarrow \frac{n}{2}+$, one has

$$
\lambda_{1} \leq n^{2} \beta
$$

The proof of the theorem is complete.

For every $\epsilon>0$, let $D_{\epsilon}$ be a subdomain of $D$ defined by

$$
D_{\epsilon}=\{z \in D: r(z)<-\epsilon\}
$$

Note that $\partial D_{\epsilon} \in C^{2}$ and $D_{\epsilon} \uparrow D$ as $\epsilon \rightarrow 0^{+}$. Then we have the following result:

Theorem 2.3. If $\lim _{z \rightarrow \partial D}|\partial u|_{u}^{2}=\beta$ and $\int_{\partial D_{t}} J(r)(z) d \sigma(z)$ is a continuous function of $t$ on $[0,1]$, then

$$
\lambda_{1}(D) \leq n^{2} \beta
$$

Proof. For $0<\epsilon_{1}<<\epsilon<1$, let $\eta=s / \alpha>0$ and let

$$
f(z)= \begin{cases}\left(-r(z)-\epsilon_{1}\right)^{\alpha}(\epsilon+r(z))^{s} & \text { if } 0<\epsilon_{1} \leq-r(z)<\epsilon \\ 0 & \text { otherwise }\end{cases}
$$

Since $\lim _{z \rightarrow \partial D}|\partial u|_{u}^{2}=\beta$ and $\lim _{t \rightarrow 0} \int_{\partial D_{t}} J(r)(z) d \sigma(z)=\int_{\partial D} J(r)(z) d \sigma(z)$, there exists $\delta(\epsilon)>0$ such that $|\partial u|_{u}^{2} \leq \beta(1+\delta(\epsilon))$ on $D \backslash D_{\epsilon}$ and $\mid \int_{\partial D_{\epsilon}}$ 
$J(r)(z) d \sigma(z)-\int_{\partial D} J(r)(z) d \sigma(z) \mid \leq \delta(\epsilon)$ with $\lim _{\epsilon \rightarrow 0^{+}} \delta(\epsilon)=0$. Thus, for $z$ $\in D \backslash D_{\epsilon}$

$$
\begin{aligned}
\sum_{i, j=1}^{n} u^{i \bar{j}} f_{i} f_{\bar{j}}= & |f(z)|^{2} \sum_{i, j=1}^{n} u^{i \bar{j}}(\log f)_{i}(\log f)_{\bar{j}} \\
= & \alpha^{2}|f(z)|^{2}\left(\frac{r}{r+\epsilon_{1}}+\eta \frac{r}{\epsilon+r}\right)^{2} \sum_{i, j=1}^{n} u^{i \bar{j}} u_{i} u_{\bar{j}} \\
\leq & \alpha^{2}(1+\delta(\epsilon)) \beta r^{2}(\epsilon+r)^{2 s-2}\left(-\epsilon_{1}-r\right)^{2(\alpha-1)} \\
& \times\left((1+\eta) r+\epsilon+\eta \epsilon_{1}\right)^{2} .
\end{aligned}
$$

Let $2 \alpha=n$ and $2 s>2$ and let $C=\int_{\partial D} J(r)(z) d \sigma(z)$. Then

$$
\begin{aligned}
& \int_{D_{\epsilon_{1}} \backslash D_{\epsilon}} \sum_{i, j=1}^{n} u^{i \bar{j}} f_{i} f_{\bar{j}} d V_{u} \\
& \leq \alpha^{2}(1+\delta(\epsilon)) \beta \int_{D_{\epsilon_{1}} \backslash D_{\epsilon}} r^{2}(\epsilon+r)^{2 s-2}\left(-\epsilon_{1}-r\right)^{2 \alpha-2} \\
& \times\left((1+\eta) r+\epsilon+\eta \epsilon_{1}\right)^{2} \operatorname{det} H(u)(z) d v(z) \\
& =\alpha^{2}(1+\delta(\epsilon)) \beta \int_{\epsilon_{1}}^{\epsilon} \int_{\partial D_{t}}(\epsilon-t)^{2 s-2}\left(-\epsilon_{1}+t\right)^{2(\alpha-1)} \\
& \times\left((1+\eta) t-\epsilon-\eta \epsilon_{1}\right)^{2} t^{-n+1} J(r) d \sigma(z) d t \\
& \leq \alpha^{2}(1+\delta(\epsilon)) \beta C \int_{\epsilon_{1}}^{\epsilon}(\epsilon-t)^{2 s-2}\left(t-\epsilon_{1}\right)^{2 \alpha-2}\left((1+\eta) t-\epsilon-\eta \epsilon_{1}\right)^{2} t^{-n+1} d t \\
& +C \delta(\epsilon) \alpha^{2}(1+\delta(\epsilon)) \beta \int_{\epsilon_{1}}^{\epsilon}(\epsilon-t)^{2 s-2}\left(t-\epsilon_{1}\right)^{2 \alpha-2} \\
& \times\left((1+\eta) t-\epsilon-\eta \epsilon_{1}\right)^{2} t^{-n+1} d t \\
& =\alpha^{2}(1+\delta(\epsilon)) \beta C \int_{\epsilon_{1}}^{\frac{\epsilon}{1+\eta}}(\epsilon-t)^{2 s-2}\left(t-\epsilon_{1}\right)^{2 \alpha-2} \\
& \times\left((1+\eta) t-\epsilon-\eta \epsilon_{1}\right)^{2} t^{-n+1} d t \\
& +\alpha^{2}(1+\delta(\epsilon)) \beta C \int_{\frac{\epsilon}{1+\eta}}^{\epsilon}(\epsilon-t)^{2 s-2}\left(t-\epsilon_{1}\right)^{2 \alpha-2} \\
& \times\left((1+\eta) t-\epsilon-\eta \epsilon_{1}\right)^{2} t^{-n+1} d t \\
& +C \delta(\epsilon) \alpha^{2}(1+\delta(\epsilon)) \beta \int_{\epsilon_{1}}^{\epsilon}(\epsilon-t)^{2 s-2}\left(t-\epsilon_{1}\right)^{2 \alpha-2} \\
& \times\left((1+\eta) t-\epsilon-\eta \epsilon_{1}\right)^{2} t^{-n+1} d t
\end{aligned}
$$


Laplace-Beltrami operator on a bounded pseudoconvex domain 385

$$
\begin{aligned}
\leq & \alpha^{2}(1+\delta(\epsilon)) \beta C \epsilon^{2 s} \int_{\epsilon_{1}}^{\frac{\epsilon}{1+\eta}} t^{-1} d t+\alpha^{2}(1+\delta(\epsilon)) \beta C\left(1+\eta^{2}\right) \epsilon^{2 s} \int_{\frac{\epsilon}{1+\eta}}^{\epsilon} t^{-1} d t \\
& +C \delta(\epsilon) \alpha^{2}(1+\delta(\epsilon)) \beta\left(1+\eta^{2}\right) \epsilon^{2 s} \int_{\epsilon_{1}}^{\epsilon} t^{-1} d t \\
\leq & C \alpha^{2}(1+\delta(\epsilon)) \beta \\
& \times\left[\epsilon^{2 s} \log \left(\frac{\epsilon}{\epsilon_{1}}\right)+\left(1+\eta^{2}\right) \epsilon^{2 s} \log (1+\eta)+\delta(\epsilon)\left(1+\eta^{2}\right) \epsilon^{2 s} \log \left(\frac{\epsilon}{\epsilon_{1}}\right)\right]
\end{aligned}
$$

and for $\epsilon_{1}<<\epsilon_{2} \leq \frac{1}{2} \epsilon$

$$
\begin{aligned}
& \int_{D_{\epsilon_{1} \backslash D_{\epsilon}}}|f(z)|^{2} d V_{u}(z) \\
& =\int_{D_{\epsilon_{1}} \backslash D_{\epsilon}}(\epsilon+r)^{2 s}\left(-\epsilon_{1}-r\right)^{2 \alpha}(-r)^{-n-1} J(r) d v(z) \\
& =\int_{\epsilon_{1}}^{\epsilon} \int_{\partial D_{t}}(\epsilon-t)^{2 s}\left(-\epsilon_{1}+t\right)^{2 \alpha} t^{-n-1} J(r) d \sigma(z) d t \\
& \geq C\left[\int_{\epsilon_{1}}^{\epsilon}(\epsilon-t)^{2 s}\left(t-\epsilon_{1}\right)^{2 \alpha} t^{-n-1} d t-\delta(\epsilon) \int_{\epsilon_{1}}^{\epsilon}(\epsilon-t)^{2 s}\left(t-\epsilon_{1}\right)^{2 \alpha} t^{-n-1} d t\right] \\
& \geq C\left[\left(\epsilon-\epsilon_{2}\right)^{2 s} \int_{\epsilon_{1}}^{\epsilon_{2}}\left(t-\epsilon_{1}\right)^{n} t^{-1-n} d t-\delta(\epsilon) \epsilon^{2 s} \int_{\epsilon_{1}}^{\epsilon} t^{-1} d t\right] \\
& =C\left[\left(\epsilon-\epsilon_{2}\right)^{2 s}\left[\sum_{k=1}^{n} C_{k}^{n}\left(-\epsilon_{1}\right)^{k} \frac{1}{k}\left(\frac{1}{\epsilon_{1}^{k}}-\frac{1}{\epsilon_{2}^{k}}\right)+\log \frac{\epsilon_{2}}{\epsilon_{1}}\right]-\delta(\epsilon) \epsilon^{2 s} \int_{\epsilon_{1}}^{\epsilon} t^{-1} d t\right] \\
& =C\left[\left(\epsilon-\epsilon_{2}\right)^{2 s}\left[\sum_{k=1}^{n} C_{k}^{n}(-1)^{k} \frac{1}{k}\left(1-\frac{\epsilon_{1}^{k}}{\epsilon_{2}^{k}}\right)+\log \left(\frac{\epsilon_{2}}{\epsilon_{1}}\right)\right]-\delta(\epsilon) \epsilon^{2 s} \log \left(\frac{\epsilon}{\epsilon_{1}}\right)\right] .
\end{aligned}
$$

Therefore, with $\epsilon_{2}=\epsilon^{2}$,

$$
\lim _{\epsilon_{1} \rightarrow 0^{+}} \frac{4 \int_{D_{\epsilon_{1}} \backslash D_{\epsilon}} u^{i \bar{j}} f_{i} f_{\bar{j}} d V_{u}}{\int_{D_{\epsilon_{1}} \backslash D_{\epsilon}}|f(z)|^{2} d V_{u}} \leq 4 \alpha^{2}\left(1+C_{0} \delta(\epsilon)\right) \beta \frac{1}{(1-\epsilon)^{2 s}-\delta(\epsilon)},
$$

where $C_{0}$ is a positive constant independent of $\epsilon$. By the domain monotonicity of eigenvalues, for any $\epsilon>0$, one has that

$$
\lambda_{1}(D) \leq \lambda_{1}\left(D \backslash D_{\epsilon}\right) \leq 4 \alpha^{2}\left(1+C_{0} \delta(\epsilon)\right) \beta \frac{1}{(1-\epsilon)^{2 s}-\delta(\epsilon)} .
$$


Let $\alpha=\frac{n}{2}$ and then let $\epsilon \rightarrow 0^{+}$. Thus, one has

$$
\lambda_{1}(D) \leq n^{2} \beta .
$$

The proof of the theorem is complete.

Now we are ready to prove Theorem 1.1.

\section{Proof of Theorem 1.1.}

Proof. Part (a) of Theorem 1.1 follows from Theorems 2.3 and 2.4.

To prove part (b) of the theorem, since $r$ is plurisubharmonic in $D$, without loss of generality, we may assume $H(r)(z)$ is positive definite for $z \in D$ - otherwise, we may use $r_{1}(z)=r(z)+\epsilon\left(|z|^{2}-A\right)$ to replace $r-$ and carry out the following computation.

By (2.9), one has that

$$
|\partial u|_{u}^{2}=\frac{|\partial r|_{r}^{2}}{-r+|\partial r|_{r}^{2}} \leq 1
$$

By Proposition 2.2, one has $\lambda_{1}(D) \geq n^{2}$. Therefore, part (b) of Theorem 1.1 follows.

Finally, by (2.9), one has

$$
|\partial u|_{u}^{2}=\frac{|\partial r|_{r}^{2}}{-r+|\partial r|_{r}^{2}}
$$

This implies that

$$
\beta(z)=\limsup _{w \rightarrow z}|\partial u(w)|_{u}^{2}=1, \quad z \in \partial D
$$

and $|\partial u|_{u}^{2} \leq 1$ on $D$. By parts (a) and (b), one has proved part (c). Therefore, the proof of Theorem 1.1 is complete.

\section{Proof of Corollary 1.2}

We are now ready to prove Corollary 1.2. 
Proof. (i) We first consider the Laplace-Beltrami operator in KählerEinstein metric. Let $u$ be strictly plurisubharmonic in $D$ so that

$$
\begin{cases}\operatorname{det} H(u)=\mathrm{e}^{(n+1) u} & \text { in } D, \\ u=+\infty & \text { on } \partial D\end{cases}
$$

and let

$$
r(z)=-\mathrm{e}^{-u(z)} .
$$

Then $J(r)=1$ and by Cheng and Yau [2], Lee and Melrose [7], one has that $r(z) \in C^{n+2-\epsilon}(\bar{D})$ for any $\epsilon>0$. Thus $\partial r \neq 0$ on $\partial D$ and

$$
\operatorname{det} H(r)=\mathrm{e}^{u}\left(1-|\partial u|_{u}^{2}\right) \quad \text { on } D .
$$

Since $\operatorname{det} H(r)(z)$ is bounded on $\bar{D}$ and $u(z) \rightarrow+\infty$ as $z \rightarrow \partial D$, this implies that

$$
\lim _{z \rightarrow \partial D}|\partial u|_{u}^{2}=1 .
$$

Applying Theorem 2.4 with $\beta=1$, one has that $\lambda_{1}(D) \leq n^{2}$.

(ii) Let $K$ be the Bergman kernel function, and let $u(z)=\frac{1}{n+1} \log K$ $(z, z)$. Then $u(z)$ is strictly plurisubharmonic in $D$. Let $r(z)=-\mathrm{e}^{-u(z)}$. Then $r \in C^{n+2-\epsilon}(\bar{D})$ is a defining function for $D$ by the result of Fefferman [4]. Let $\rho \in C^{\infty}(\bar{D})$ be any strictly plurisubharmonic defining function for $D$. By Fefferman [4], one has that

$$
u(z)=-\log (-\rho(z))+b(z),
$$

where $b \in C^{n+2-\epsilon}(\bar{D})$. Then

$$
\left[u^{i \bar{j}}\right]=\left[(-\log (-\rho))^{i \bar{j}}\right]\left(I_{n}+\rho B\right),
$$

where $B$ is an $n \times n$ matrix with bounded entries near $\partial D$. Let $u^{0}=$ $-\log (-\rho)$. Then by $(2.9), \lim _{z \rightarrow \partial D}\left|\partial u^{0}\right|_{u^{0}}^{2}=1$. It is easy to see that

$$
\left|\partial u^{0}\right|_{u^{0}}^{2}(1+C \rho) \leq|\partial u|_{u}^{2} \leq\left|\partial u^{0}\right|_{u^{0}}^{2}(1-C \rho)
$$

for some $C>>1$ and $z$ near $\partial D$. Therefore, $\lim _{z \rightarrow \partial D}|\partial u|_{u}^{2}=1$. Applying Theorem 2.4 with $\beta=1$, one has that $\lambda_{1}(D) \leq n^{2}$ for the LaplaceBeltrami operator in Bergman metric. Therefore, the proof of Corollary 1.2 is complete. 


\section{Weakly pseudoconvex domains with $\lambda_{1}=n^{2}$}

In this section, we consider the complex ellipsoid in $\mathbb{C}^{2}(n=2)$

$$
E_{m}=\left\{\left(z_{1}, z_{2}\right):\left|z_{2}\right|^{2 m}+\left|z_{1}\right|^{2}<1\right\}, \quad m>1
$$

which is a weakly (not strongly) pseudoconvex domain in $\mathbb{C}^{2}$. We let

$$
r(z)=\left|z_{2}\right|^{2}-\left(1-\left|z_{1}\right|^{2}\right)^{1 / m}
$$

Then $r(z) \in C^{\infty}\left(E_{m}\right) \cap C^{1 / m}\left(E_{m}\right)$ is a strictly plurisubharmonic defining function for $E_{m}$ (see [14] for more details) with

$$
H(r)=\left[\begin{array}{cc}
\frac{\left(1-\left|z_{1}\right|^{2}\right)^{1 / m-2}}{m^{2}}\left(m-\left|z_{1}\right|^{2}\right) & 0 \\
0 & 1
\end{array}\right] .
$$

Let

$$
u(z)=-\log (-r(z)), \quad z \in E_{m}
$$

Then the following holds:

Proposition 4.1. Let $\Delta_{u}$ be the Laplace-Beltrami operator associated to the Kähler metric $u_{i \bar{j}} d z_{i} \otimes d \bar{z}_{j}$ on $E_{m}$. Then $\lambda_{1}\left(\Delta_{u}\right)=4=2^{2}$.

Proof. By (4.3), one has

$$
\begin{aligned}
|\partial r|_{r}^{2} & =\frac{m\left(1-\left|z_{1}\right|^{2}\right)^{2-1 / m}}{1-\frac{\left|z_{1}\right|^{2}}{m}} \frac{1}{m^{2}}\left(1-\left|z_{1}\right|^{2}\right)^{2 / m-2}\left|z_{1}\right|^{2}+\left|z_{2}\right|^{2} \\
& =\frac{\left(1-\left|z_{1}\right|^{2}\right)^{1 / m}}{m-\left|z_{1}\right|^{2}}\left|z_{1}\right|^{2}+\left|z_{2}\right|^{2}
\end{aligned}
$$

$$
\begin{aligned}
|\partial r|_{r}^{2}-r(z) & =\left(1-\left|z_{1}\right|^{2}\right)^{1 / m}\left(1+\frac{\left|z_{1}\right|^{2}}{m-\left|z_{1}\right|^{2}}\right) \\
& =\frac{m\left(1-\left|z_{1}\right|^{2}\right)^{1 / m}}{m-\left|z_{1}\right|^{2}}
\end{aligned}
$$


Laplace-Beltrami operator on a bounded pseudoconvex domain 389

$$
\begin{aligned}
|\partial u|_{u}^{2} & =\frac{|\partial r|_{r}^{2}}{|\partial r|_{r}^{2}-r}=\frac{m\left|z_{2}\right|^{2}-r(z)\left|z_{1}\right|^{2}}{m\left(1-\left|z_{1}\right|^{2}\right)^{1 / m}} \\
& =1+r(z) \frac{m-\left|z_{1}\right|^{2}}{m\left(1-\left|z_{1}\right|^{2}\right)^{1 / m}}
\end{aligned}
$$

and

$$
\begin{aligned}
J(r)= & \operatorname{det} H(r)\left[|\partial r|_{r}^{2}-r\right] \\
= & \frac{\left(1-\left|z_{1}\right|^{2}\right)^{1 / m-2}}{m}\left[1-\frac{\left|z_{1}\right|^{2}}{m}\right] \\
& \times\left[\left(1-\left|z_{1}\right|^{2}\right)^{1 / m}+\frac{\left(1-\left|z_{1}\right|^{2}\right)^{1 / m}}{m-\left|z_{1}\right|^{2}}\left|z_{1}\right|^{2}\right] \\
= & \frac{\left(1-\left|z_{1}\right|^{2}\right)^{2 / m-2}}{m} .
\end{aligned}
$$

Moreover,

$$
r^{1 \overline{1}}=\frac{m^{2}}{m-\left|z_{1}\right|^{2}}\left(1-\left|z_{1}\right|^{2}\right)^{2-1 / m}, \quad r^{2 \overline{2}}=1, \quad r^{1 \overline{2}}=0
$$

and

$$
r^{1}=\frac{m}{m-\left|z_{1}\right|^{2}}\left(1-\left|z_{1}\right|^{2}\right) z_{1}, \quad r^{2}=z_{2} .
$$

Therefore,

$$
\begin{aligned}
{\left[u^{i \bar{j}}\right] } & =(-r(z))\left[r^{i \bar{j}}-\frac{r^{i} r^{\bar{j}}}{|\partial r|_{r}^{2}-r}\right] \\
& =(-r(z))\left[\begin{array}{cc}
m\left(1-\left|z_{1}\right|^{2}\right)^{2-1 / m} & -\left(1-\left|z_{1}\right|^{2}\right)^{1-1 / m} z_{1} \bar{z}_{2} \\
-\left(1-\left|z_{1}\right|^{2}\right)^{1-1 / m} z_{2} \bar{z}_{1} & 1-\frac{\left(m-\left|z_{1}\right|^{2}\right)}{m} \frac{\left|z_{2}\right|^{2}}{\left(1-\left|z_{1}\right|^{2}\right)^{1 / m}}
\end{array}\right]
\end{aligned}
$$

Let

$$
h(z)=\beta \log \left(1-\left|z_{1}\right|^{2}\right) \quad \text { and } \quad f=\mathrm{e}^{-\alpha u+h},
$$


where $\alpha$ and $\beta$ are positive constants. Then

$$
\begin{aligned}
& \sum_{i, j=1}^{2} u^{i \bar{j}} u_{i} h_{\bar{j}} \\
& =\beta\left[m\left(1-\left|z_{1}\right|^{2}\right)^{2-1 / m} \frac{1}{m}\left(1-\left|z_{1}\right|^{2}\right)^{1 / m-1} \bar{z}_{1}-\left(1-\left|z_{1}\right|^{2}\right)^{1-1 / m} z_{2} \bar{z}_{1} \bar{z}_{2}\right] \\
& \quad \times\left[\frac{-z_{1}}{1-\left|z_{1}\right|^{2}}\right] \\
& =-\beta\left|z_{1}\right|^{2}\left(1-\frac{\left|z_{2}\right|^{2}}{\left(1-\left|z_{1}\right|^{2}\right)^{1 / m}}\right) \\
& =-\beta\left|z_{1}\right|^{2}\left(1-\left|z_{1}\right|^{2}\right)^{-1 / m}(-r(z))
\end{aligned}
$$

and

$$
\begin{aligned}
\sum_{i, j=1}^{2} u^{i \bar{j}} h_{i \bar{j}} & =m \beta r\left(1-\left|z_{1}\right|^{2}\right)^{1-1 / m}\left[\left(1-\left|z_{1}\right|^{2}\right)^{-1}\left|z_{1}\right|^{2}+1\right] \\
& =m \beta r\left(1-\left|z_{1}\right|^{2}\right)^{-1 / m}\left|z_{1}\right|^{2}+m \beta r\left(1-\left|z_{1}\right|^{2}\right)^{1-1 / m}
\end{aligned}
$$

and

$$
\begin{aligned}
\sum_{i, j=1}^{2} u^{i \bar{j}} h_{i} h_{\bar{j}} & =(-r) m\left(1-\left|z_{1}\right|^{2}\right)^{2-1 / m} \beta^{2}\left(1-\left|z_{1}\right|^{2}\right)^{-2}\left|z_{1}\right|^{2} \\
& =m \beta^{2}(-r)\left|z_{1}\right|^{2}\left(1-\left|z_{1}\right|^{2}\right)^{-1 / m}
\end{aligned}
$$

Thus

$$
\begin{aligned}
\mathrm{e}^{\alpha u-h} \Delta_{u}\left(\mathrm{e}^{-\alpha u+h}\right) & \\
= & 4 \alpha n-4 \alpha^{2}|\partial u|_{u}^{2}-4 \sum_{i, j=1}^{2} u^{i \bar{j}}\left(h_{i \bar{j}}+h_{i} h_{\bar{j}}\right)+4 \alpha 2 \operatorname{Re} \sum_{i, j=1}^{2} u^{i \bar{j}} u_{i} h_{\bar{j}} \\
= & 4 \alpha(n-\alpha)+4 \alpha^{2} \frac{-r(z)}{\left(1-\left|z_{1}\right|^{2}\right)^{1 / m}} \frac{m-\left|z_{1}\right|^{2}}{m} \\
& +4 m \beta \frac{-r(z)}{\left(1-\left|z_{1}\right|^{2}\right)^{1 / m}}\left(1-\beta\left|z_{1}\right|^{2}\right)-8 \alpha \beta\left|z_{1}\right|^{2}\left(1-\left|z_{1}\right|^{2}\right)^{-1 / m}(-r(z)) \\
= & 4 \alpha(n-\alpha)+4 \frac{-r(z)}{\left(1-\left|z_{1}\right|^{2}\right)^{1 / m}}\left[\beta m\left(1-\beta\left|z_{1}\right|^{2}\right)-2 \beta \alpha\left|z_{1}\right|^{2}+\alpha^{2} \frac{m-\left|z_{1}\right|^{2}}{m}\right]
\end{aligned}
$$


Laplace-Beltrami operator on a bounded pseudoconvex domain 391

$$
=4 \alpha(n-\alpha)+4 \frac{-r(z)}{\left(1-\left|z_{1}\right|^{2}\right)^{1 / m}}\left[\beta m+\alpha^{2}-\left(m \beta^{2}+2 \beta \alpha+\frac{\alpha^{2}}{m}\right)\left|z_{1}\right|^{2}\right]
$$

and

$$
\begin{aligned}
\mathrm{e}^{2(\alpha u-h)}\left|\partial\left(\mathrm{e}^{-\alpha u+h}\right)\right|_{u}^{2} \\
=\alpha^{2}|\partial u|_{u}^{2}-\alpha u^{i \bar{j}}\left(u_{i} h_{\bar{j}}+u_{\bar{j}} h_{i}\right)+u^{i \bar{j}} h_{i} h_{\bar{j}} \\
=\alpha^{2}-\alpha^{2} \frac{-r(z)}{\left(1-\left|z_{1}\right|^{2}\right)^{1 / m}} \frac{m-\left|z_{1}\right|^{2}}{m} \\
\quad+m \beta^{2} \frac{-r(z)}{\left(1-\left|z_{1}\right|^{2}\right)^{1 / m}}\left|z_{1}\right|^{2}+2 \alpha \beta\left|z_{1}\right|^{2}\left(1-\left|z_{1}\right|^{2}\right)^{-1 / m}(-r(z)) \\
\quad \alpha^{2}+\frac{-r(z)}{\left(1-\left|z_{1}\right|^{2}\right)^{1 / m}}\left[-\alpha^{2} \frac{m-\left|z_{1}\right|^{2}}{m}+m \beta^{2}\left|z_{1}\right|^{2}+2 \alpha \beta\left|z_{1}\right|^{2}\right] \\
=\alpha^{2}+\frac{-r(z)}{\left(1-\left|z_{1}\right|^{2}\right)^{1 / m}}\left[-\alpha^{2}+\frac{\left|z_{1}\right|^{2}}{m}\left(\alpha^{2}+m^{2} \beta^{2}+2 m \alpha \beta\right)\right] .
\end{aligned}
$$

Let $2 \alpha>n$ and $2 \beta=1$ with $n=2$. Then

$$
\begin{aligned}
m & \int_{E_{m}}|f(z)|^{2}(-r(z))^{-n-1} J(r) d v(z) \\
= & \int_{\left|z_{1}\right|<1} \int_{\left|z_{2}\right|^{2}<\left(1-\left|z_{1}\right|^{2}\right)^{1 / m}}\left(\left(1-\left|z_{1}\right|^{2}\right)^{1 / m}-\left|z_{2}\right|^{2}\right)^{2 \alpha-n-1} \\
& \times\left(1-\left|z_{1}\right|^{2}\right)^{2 / m+2 \beta-2} d A\left(z_{2}\right) d A\left(z_{1}\right) \\
= & \pi \int_{\left|z_{1}\right|<1}\left(1-\left|z_{1}\right|^{2}\right)^{2 / m+2 \beta-2} \\
& \times \int_{0}^{\left(1-\left|z_{1}\right|^{2}\right)^{1 / m}}\left(\left(1-\left|z_{1}\right|^{2}\right)^{1 / m}-t\right)^{2 \alpha-n-1} d t d A\left(z_{1}\right) \\
= & \pi \int_{\left|z_{1}\right|<1}\left(1-\left|z_{1}\right|^{2}\right)^{2 / m+2 \beta-2} \frac{1}{2 \alpha-n}\left(\left(1-\left|z_{1}\right|^{2}\right)^{1 / m}\right)^{2 \alpha-n} d A\left(z_{1}\right) \\
= & \frac{\pi}{2 \alpha-n} \int_{\left|z_{1}\right|<1}\left(1-\left|z_{1}\right|^{2}\right)^{\frac{2+2 \alpha-n}{m}+2 \beta-2} d A\left(z_{1}\right) \\
= & \frac{\pi^{2}}{2 \alpha-n} \int_{0}^{1}(1-t)^{\frac{2 \alpha}{m}+2 \beta-2} d t \\
= & \frac{m \pi^{2}}{(2 \alpha-n) 2 \alpha} .
\end{aligned}
$$


Moreover,

$$
\begin{aligned}
& m \int_{E_{m}}|f(z)|^{2} \frac{(-r(z))}{\left(1-\left|z_{1}\right|^{2}\right)^{1 / m}} \\
& \times \quad\left[-\alpha^{2}+\frac{\left|z_{1}\right|^{2}}{m}\left(\alpha^{2}+m^{2} \beta^{2}+2 m \alpha \beta\right)\right](-r(z))^{-n-1} J(r)(z) d v(z) \\
& =\int_{\left|z_{1}\right|<1} \int_{\left|z_{2}\right|^{2}<\left(1-\left|z_{1}\right|^{2}\right)^{1 / m}}\left(\left(1-\left|z_{1}\right|^{2}\right)^{1 / m}-\left|z_{2}\right|^{2}\right)^{2 \alpha-n} \\
& \times \frac{\left(\alpha^{2}+m^{2} / 4+m \alpha\right)\left|z_{1}\right|^{2}}{m\left(1-\left|z_{1}\right|^{2}\right)^{1-1 / m}} d A\left(z_{2}\right) d A\left(z_{1}\right) \\
& -\int_{\left|z_{1}\right|<1} \int_{\left|z_{2}\right|^{2}<\left(1-\left|z_{1}\right|^{2}\right)^{1 / m}}\left(\left(1-\left|z_{1}\right|^{2}\right)^{1 / m}-\left|z_{2}\right|^{2}\right)^{2 \alpha-n} \\
& \times \frac{\alpha^{2}}{\left(1-\left|z_{1}\right|^{2}\right)^{1-1 / m}} d A\left(z_{2}\right) d A\left(z_{1}\right) \\
& =\frac{\pi}{2 \alpha-1} \int_{\left|z_{1}\right|<1}\left(1-\left|z_{1}\right|^{2}\right)^{\frac{2 \alpha}{m}-1}\left(\alpha^{2} / m+m / 4+\alpha\right)\left|z_{1}\right|^{2} d A\left(z_{1}\right) \\
& -\frac{\pi \alpha^{2}}{2 \alpha-1} \int_{\left|z_{1}\right|<1}\left(1-\left|z_{1}\right|^{2}\right)^{\frac{2 \alpha}{m}-1} d A\left(z_{1}\right) \\
& =\frac{\pi^{2}}{2 \alpha-1} \int_{0}^{1}(1-t)^{\frac{2 \alpha}{m}-1}\left(\alpha^{2} / m+m / 4+\alpha\right) t d t-\frac{\alpha^{2} \pi^{2}}{2 \alpha-1} \frac{m}{2 \alpha} \\
& =\frac{\pi^{2}}{(2 \alpha-1) 2 \alpha} \int_{0}^{1}(1-t)^{\frac{2 \alpha}{m}}\left(\alpha^{2}+m^{2} / 4+m \alpha\right) d t-\frac{\alpha m \pi^{2}}{2(2 \alpha-1)} \\
& =\frac{\pi^{2} m}{(2 \alpha-1) 2 \alpha(2 \alpha+m)}\left(\alpha^{2}+m^{2} / 4+m \alpha\right)-\frac{\alpha m \pi^{2}}{2(2 \alpha-1)} \text {. }
\end{aligned}
$$

So (4.13) implies that

$$
\begin{aligned}
& m \int_{E_{m}}|\nabla f(z)|^{2}(-r(z))^{-n-1} J(r) d v(z) \\
& =4 \alpha^{2} m \int_{E_{m}}|f(z)|^{2}(-r(z))^{-n-1} J(r) d v(z) \\
& \quad+4\left[\frac{\pi^{2} m}{(2 \alpha-1) 2 \alpha(2 \alpha+m)}\left(\alpha^{2}+m^{2} / 4+m \alpha\right)-\frac{\alpha m \pi^{2}}{2(2 \alpha-1)}\right] .
\end{aligned}
$$


Laplace-Beltrami operator on a bounded pseudoconvex domain 393

Thus

$$
\begin{aligned}
\lambda_{1} \leq & 4 \alpha^{2}+4 \frac{(2 \alpha-2) 2 \alpha}{m \pi^{2}} \\
& \times\left[\frac{\pi^{2} m}{(2 \alpha-1) 2 \alpha(2 \alpha+m)}\left(\alpha^{2}+\frac{m^{2}}{4}+m \alpha\right)-\frac{\alpha m \pi^{2}}{2(2 \alpha-1)}\right] \\
= & 4 \alpha^{2}+4\left[\frac{(\alpha+m / 2)}{(2 \alpha-1) \alpha 2}-\frac{\alpha}{(2 \alpha-1)}\right](2 \alpha-2) \alpha .
\end{aligned}
$$

Letting $2 \alpha \rightarrow 2^{+}$, we have

$$
\lambda_{1} \leq 4
$$

On the other hand, since $|\partial u|_{u}^{2} \leq 1$ in $E_{m}$, Proposition 2.2 implies that

$$
\lambda_{1} \geq 2^{2}=4 .
$$

Therefore, $\lambda_{1}=2^{2}$, and the proof of the proposition is complete.

Next we will make a remark and pose a question. Note that

$$
\begin{aligned}
\log \operatorname{det} H(u)(z) & =(n+1) u+\log J(r)(z) \\
& =(n+1) u-2 \frac{m-1}{m} \log \left(1-\left|z_{1}\right|^{2}\right)-\log m
\end{aligned}
$$

where $n=2$ and $m \geq 1$, one has the Ricci curvature

$$
\begin{aligned}
R_{i \bar{j}} & =-\frac{\partial^{2} \log \operatorname{det} H(u)(z)}{\partial z_{i} \partial \bar{z}_{j}} \\
& =-(n+1) u_{i \bar{j}}+\frac{2(m-1)}{m} \frac{-1}{\left(1-\left|z_{1}\right|^{2}\right)^{2}} \delta_{1 i} \delta_{1 j},
\end{aligned}
$$

where $n=2$ and $m \geq 1$. This leads to the following remark and question.

Remark 4.1. (i) $R_{i \bar{j}} \leq-(n+1) g_{i \bar{j}}$ on $E_{m}$;

(ii) $R_{i \bar{j}} \geq-(n+1) g_{i \bar{j}}$ if and only if $m=1$ and $E_{m}=B_{2}$.

Problem 4.1. Let $D$ be a smoothly bounded pseudoconvex domain in $\mathbb{C}^{n}$ with a negative defining function $r(z)$ so that $u(z)=-\log (-r(z))$ is strictly plurisubharmonic in $D$ induced a Kähler metric $u=\sum_{i, j=1}^{n} u_{i j} d z_{i} \otimes d \bar{z}_{j}$. Assume $R_{i \bar{j}} \geq-(n+1) g_{i \bar{j}}$ on $(D, u)$ and $\lambda_{1}=n^{2}$. What can one say about D? 


\section{Acknowledgment}

M.-A.T. was partially supported by MS-0801988

\section{References}

[1] S. Y. Cheng, Eigenvalue comparison theorems and its geometric application, Math. Z. 143 (1975), 289-297.

[2] S.-Y. Cheng and S.-T. Yau, On the existence of a complex Kähler metric on noncompact complex manifolds and the regularity of Fefferman's equation, Comm. Pure Appl. Math. 33 (1980), 507-544.

[3] C. Fefferman, Monge-Ampère equations, the Bergman kernel, and geometry of pseudoconvex domains, Ann. of Math. 103 (1976), 395-416.

[4] C. Fefferman, The Bergman kernel and biholomorphic mappings of pseudoconvex domains, Invent. Math. 65 (1974), 1-65.

[5] L. Ji, P. Li and J. Wang, Ends of locally symmetric spaces with maximal bottom spectrum, J. Reine Angew. Math. 632 (2009), 1-35. 58Jxx (22Exx)

[6] S. Kong, P. Li and D. Zhou, Spectrum of the Laplacian on quaternionic Kähler manifolds, J. Differential Geom. 78 (2008), 295-332.

[7] J.M. Lee and R. Melrose, Boundary behavior of the complex MongeAmpère equation, Acta Math. 148 (1982), 159-192.

[8] P. Li, Lecture notes on geometric analysis, Lecture Notes Series, 6, Research Institute of Mathematics and Global Analysis Research Center, Seoul National University, Korea, 1993.

[9] P. Li, Harmonic functions on complete Riemannian manifolds, Handbook of Geometric Analysis, No. 1, Advanced Lectu. Maths, 7, International Press, 2008.

[10] P. Li and J. Wang, Comparison theorem for Kähler manifolds and positivity of spectrum, J. Differential Geom. 69 (2005), 43-74.

[11] P. Li and J. Wang, Complete manifolds with positive spectrum II, J. Differential Geom. 62 (2002), 143-162.

[12] P. Li and J. Wang, Complete manifolds with positive spectrum, J. Differential Geom. 58 (2001), 501-534. 
Laplace-Beltrami operator on a bounded pseudoconvex domain 395

[13] S.-Y. Li, Characterization for balls by potential function of KählerEinstein metrics for domains in $\mathbb{C}^{n}$, Comm. Anal. Geom. 13(2) (2005), 461-478.

[14] S.-Y. Li, On the existence and regularity of Dirichlet problem for complex Monge-Ampère equations on weakly pseudoconvex domains, Calc. Var. Partial Differential Equations 20 (2004), 119-132.

[15] S.-Y. Li, Characterization for a class of pseudoconvex domains whose boundaries having positive constant pseudo scalar curvature, CAG 17 (2009), 17-35.

[16] O. Munteanu, A sharp estimate for the bottom of the spectrum of the Laplacian on Kähler manifolds, J. Differential Geom. 83 (2009), 163187.

[17] S. Udagawa, Compact Kähler manifolds and the eigenvalues of the Laplacian, Colloq. Math. 56(2) (1988), 341-349.

Department of Mathematics

UNIVERSITY OF CALIFORNIA

IRVINE

CA 92697-3875, USA

E-mail address: sli@math.uci.edu

E-mail address: mtran@math.uci.edu

ReCEIVED OCTOBER 30, 2009 
